\title{
Reversible digital clubbing in acute myeloid leukaemia
}

\author{
S. Bhandari, M.A. Wodzinski and J.T. Reilly \\ Department of Haematology, Northern General Hospital, Sheffield S5 7AU, UK
}

\begin{abstract}
Summary: A 43 year old woman presented with acute myeloid leukaemia, marked finger and toe clubbing, and a hilar mass. Biopsy of the hilar mass was not technically possible, but it almost certainly represented a granulocytic sarcoma since chemotherapy induced rapid resolution of the mass along with reversal of the clubbing and remission of the leukaemia. Relapse of the leukaemia 21 months later was associated with return of the clubbing. It is hypothesized that an abnormal circulation within the granulocytic sarcoma may have accounted for the development of clubbing.
\end{abstract}

\section{Introduction}

Acquired finger clubbing is commonly associated with bronchial carcinoma, pleural tumours and other intrathoracic tumours which account for $80 \%, 10 \%$ and $5 \%$ of cases, respectively. ${ }^{1}$ Following successful treatment of the primary disorder, finger clubbing has been known to reverse. ${ }^{2-4}$ Finger clubbing has not been previously reported in association with acute myeloid leukaemia but two cases associated with chronic lymphocytic leukaemia have been documented. ${ }^{3,5} \mathrm{~A}$ case of acute myeloid leukaemia with marked finger and toe clubbing, and a likely intrathoracic granulocytic sarcoma at presentation is described.

\section{Case report}

A 43 year old woman presented with a 2 week history of malaise, intermittent central chest pain, dry cough and drenching night sweats. She had noticed a dramatic change in the shape of her finger nails over the previous 3 months. She had smoked 25 cigarettes daily for the previous 5 years.

On examination she was pyrexial and had obvious finger and toe clubbing. A blood count showed a haemoglobin of $9.8 \mathrm{~g} / \mathrm{dl}$, white cell count $2.5 \times 10^{9} / 1$ (blasts $1.4 \times 10^{9} / 1$, some with Auer rods), platelets $320 \times 10^{9} / 1$. The diagnosis of acute myeloid leukaemia FAB $^{6}$ type $\mathrm{M} 1$ was confirmed by bone marrow aspirate cytology and immunophenotyping. Cytogenetic analysis of the marrow showed 46,XX,NAD.

Chest X-ray showed a prominent left hilum, in contrast to a normal examination 3 months

Correspondence: M.A. Wodzinski, M.R.C.P., M.R.C. Path.

Accepted: 3 November 1993 previously. Sputum cytology was negative for malignant cells. Bronchoscopy showed narrowing of the left main, upper lobe, and lower lobe bronchi by external compression with no endobronchial lesion visible. Enhanced computerized tomography (CT) of the chest showed a diffuse mass in the aortopulmonary window. The proximity of the mass to major vessels precluded histological diagnosis.

She was treated with chemotherapy according to the Medical Research Council's AML 10 protocol and achieved complete remission after a course of daunorubicin, cytarabine and etoposide. This was accompanied by resolution of her finger and toe clubbing, and the left hilar mass (confirmed by CT). She received consolidation chemotherapy with daunorubicin, cytarabine and etoposide, followed by amsacrine, cytarabine and etoposide.

The leukaemia relapsed 21 months after initial diagnosis. Initially there was no evidence of finger or toe clubbing, and there was no hilar mass on chest X-ray. The leukaemia proved resistant to further intensive chemotherapy and a few weeks later she developed marked finger clubbing and died. Permission for autopsy was refused.

\section{Discussion}

The current case of acute myeloid leukaemia had marked finger clubbing associated with a likely intrathoracic hilar granulocytic sarcoma at presentation. Granulocytic sarcomas are a well-recognized complication of acute myeloid leukaemia and occur in just under $1 \%$ of cases ${ }^{7}$ and have been reported in most parts of the body. ${ }^{8}$ Two cases of mediastinal granulocytic sarcomas have been described in association with acute myeloid leukaemia but finger clubbing was not reported., ${ }^{9,10}$ Although 
histological confirmation was technically not possible, it is likely that the mediastinal mass in the present case was a granulocytic sarcoma because both the hilar mass and digital clubbing resolved when leukaemic remission was achieved with chemotherapy.

There have been a number of explanations for clubbing of the fingers. The most popular theory invokes a circulating vasodilator, such as plateletderived growth factor, which is normally inactivated by the lungs. ${ }^{11}$ This would explain the clubbing seen in the right to left cardiac shunts, and with tumours and inflammatory lesions in the lung. We hypothesize that our patient had an abnormal circulation within the granulocytic sarcoma and

\section{References}

1. Coury, C. Hippocratic fingers and hypertrophic osteoarthropathy. A study of 350 cases. Br J Dis Chest 1960, 54: 202.

2. Atkinson, M.K, McElwain, T.J., Peckham, M.J. \& Thomas, P.R.M. Hypertrophic pulmonary osteoarthropathy in Hodgkin's disease. Reversal with chemotherapy. Cancer 1976, 38: 1729-1734.

3. Glatt, W. \& Weinstein, A. Acropachy in lymphatic leukaemia. Radiology 1955, 10: 545-549.

4. Papavasiliou, C., Pavlatou, M. \& Pappas, J. Nasopharyngeal cancer in patients under the age of thirty years. Cancer 1977, 40: $2312-2316$.

5. Calvert, R.J. \& Smith, E. Metastatic acropathy in lymphatic leukemia. Blood 1955, 10: 545-549.

6. Bennett, J.M., Catovsky, D., Daniel, M.T. et al. Proposed revised criteria for the classification of acute myeloid leukemia. A report of the French-American-British Cooperative Group. Ann Intern Med 1985, 103: 626-629. this could account for the development of the $\frac{3}{3}$ digital clubbing. Clubbing recurred soon after $\stackrel{Q}{C}$ relapse of the leukaemia but, although there was no. obvious mass on chest X-ray, the presence of a hilar granulocytic sarcoma at relapse could not be excluded as autopsy was refused.

The current case demonstrates that the presence of acquired digital clubbing associated with hilar mass is not necessarily indicative of bronchogenic carcinoma in a newly diagnosed case of acute myeloid leukaemia. Chemotherapy may not only $\vec{\circ}$ lead to remission of the leukaemia, but also resolution of the clubbing and hilar mass, and ${ }_{\circ}$ should be administered in cases where biopsy is not possible.

7. Swirsky, D.M., Yi, Y.S., Matthews, J.G., Flemans, R.J., G Rees, J.K.H. \& Hayhoe, F.G.J. 8;21 translocation in acute granulocytic leukaemia: cytological, cytochemical and clinical features. Br J Haematol 1984, 56: 199-213.

8. Neiman, R.S., Barcos, M., Berard, C. et al. Granulocytic sarcoma: a clinicopathologic study of 61 biopsied cases. Cancer 1981, 48: 1426-1437.

9. Garaventa, A., Dallorso, S., Savioli, C., Rosanda, C. \& De Bernardi, B. Granulocytic sarcoma presenting as an isolated mediastinal mass. A difficult diagnostic problem. Acta Paediatr Scand 1989, 78: 473-475.

10. Muss, H.B. \& Moloney, W.C. Chloroma and other myeloblastic tumours. Blood 1973, 42: 721-728.

11. Dickinson, C.J. \& Martin, J.F. Megakaryocytes and platelet clumps as the cause of finger clubbing. Lancet 1987, ii: 1434-1435. 